\title{
Taming Nonrenormalizability
}

\author{
John R. Klauder* \\ Department of Physics and Department of Mathematics \\ University of Florida \\ P.O. Box 118440 \\ Gainesville, FL 32611-8440
}

\begin{abstract}
Nonrenormalizable scalar fields, such as $\varphi_{n}^{4}, n \geq 5$, require infinitely many distinct counter terms when perturbed about the free theory, and lead to free theories when defined as the continuum limit of a lattice regularized theory restricted only to arbitrary mass and coupling constant renormalization. Based on the proposal that functional integrals for interacting nonrenormalizable models do not reduce to the expression for the free field functional integral as the coupling constant vanishes - a proposal supported by the fact that even the set of classical solutions for such models does not reduce to the set of free field solutions as the coupling constant vanishes - it has been conjectured that for nonrenormalizable models the interaction term acts partially as a hard core eliminating certain fields otherwise allowed by the free theory. As a consequence, interacting models are continuously connected to a pseudofree theory that takes into account the hard core as the coupling constant vanishes, and this general view is supported not only by simple quantum mechanical examples as well as soluble but nonrelativistic nonrenormalizable models. The present article proposes a pseudofree model for relativistic nonrenormalizable models about which it is argued that a perturbation expansion of the interaction is term-by-term divergence free.
\end{abstract}

*klauder@phys.ufl.edu 


\section{Introduction}

Nonrenormalizable quantum field models, such as $\varphi_{n}^{4}$, with a spacetime dimension $n \geq 5$, require the introduction of nonclassical (i.e., $\hbar$-dependent), nontrivial (i.e., other than mass or interaction) counterterms to avoid triviality [1]. A conventional regularized perturbation analysis introduces additional counterterms designed to cancel divergences as they arise in the perturbative evaluation of a functional integral about the free theory; but for such theories, infinitely many distinct counterterms are needed, and therefore this approach is unsatisfactory. Instead, our procedure chooses the (unconventional) counterterm in order to cancel the source of the divergences as already identified within the integrand of a functional integral. Unconventional counterterms are not out of place since it is highly likely that interacting nonrenormalizable theories reduce to a pseudofree model different from the free model as the coupling constant is reduced to zero due to the interaction term partially acting as a hard core. Indeed, consider the Sobolev-like inequality [2]

$$
\left\{\int \phi(x)^{4} d^{n} x\right\}^{1 / 2} \leq C \int\left\{[\nabla \phi(x)]^{2}+m^{2} \phi(x)^{2}\right\} d^{n} x,
$$

valid for $C=4 / 3$ for $n \leq 4$, while $C=\infty$ for $n \geq 5$ - which means in the latter case that there are fields, e.g., $\phi_{\text {singular }}(x)=|x|^{-p} e^{-x^{2}}, n / 4 \leq p<$ $n / 2-1$, for which the left side diverges while the right side is finite. This relation implies that the set of interacting classical solutions does not reduce to the set of free classical solutions as the coupling constant goes to zero. Even simpler: the classical action for a single degree of freedom given by

$$
I=\int\left\{\frac{1}{2}\left[\dot{x}(t)^{2}-x(t)^{2}\right]-\lambda x(t)^{-4}\right\} d t
$$

clearly illustrates the basic principles of a far simpler but analogous hard-core behavior and associated pseudofree theory [3].

We turn to an analysis of the principal subject of this article. Initially, we choose an $n$-dimensional, periodic, hypercubic, Euclidean spacetime lattice with a lattice spacing $a, L$ lattice points on each side, and lattice points labeled by multi-integers $k=\left(k_{0}, k_{1}, \ldots, k_{s}\right) \in \mathbb{Z}^{n}$, where $s=n-1$ is the spatial dimension, and $k_{0}$ refers to a future time direction. The lattice-regularized functional integral for the Schwinger function generating functional is given 
by

$$
\begin{aligned}
S(h) \equiv M \int \exp \left[Z^{-1 / 2} \Sigma_{k} h_{k} \phi_{k} a^{n} / \hbar-I_{n}(\phi, a, N) / \hbar\right. \\
-C(\phi, a, \hbar) / \hbar] \Pi_{k} d \phi_{k} \\
\equiv\left\langle e^{Z^{-1 / 2} \Sigma_{k} h_{k} \phi_{k} a^{n} / \hbar}\right\rangle,
\end{aligned}
$$

where $\left\{h_{k}\right\}$ is a suitable smooth sequence, and the normalization factor $M$ ensures that $S(0)=1$. The continuum limit is taken in two steps: (i) The number of lattice sites on an edge $L \rightarrow \infty$ and the lattice spacing $a \rightarrow 0$ so that $L a$ remains constant and finite. Thus the spacetime volume $V=(L a)^{n}$ as well as the spatial volume (at fixed Euclidean time) $V^{\prime}=(L a)^{s}$ are both finite; (ii) The final step involves $V \rightarrow \infty$ and $V^{\prime} \rightarrow \infty$. In this article we focus on just the first step in the continuum limit and assume that both $V$ and $V^{\prime}$ are sufficiently large. Notationally, we also introduce $N=L^{n}$ and $N^{\prime}=L^{s}$, and note that sums (and products) such as $\Sigma_{k}\left(\Pi_{k}\right)$ are over all spacetime, while $\Sigma_{k}^{\prime}\left(\Pi_{k}^{\prime}\right)$ are over all space alone at some fixed $k_{0}$.

In (3), $Z$ denotes the field strength renormalization factor and $I_{n}(\phi, a, N)$ is the naive lattice action,

$$
I_{n}(\phi, a, N) \equiv \frac{1}{2} \sum_{k} \sum_{k^{*}}\left(\phi_{k^{*}}-\phi_{k}\right)^{2} a^{n-2}+\frac{1}{2} m_{0}^{2} \sum_{k} \phi_{k}^{2} a^{n}+\lambda_{0} \sum_{k} \phi_{k}^{4} a^{n},
$$

where $k^{*}$ denotes any one of the $n$ nearest neighbors to $k$ in the positive sense, i.e., $k^{*} \in\left\{\left(k_{0}+1, k_{1}, \ldots, k_{s}\right), \ldots,\left(k_{0}, k_{1}, \ldots, k_{s}+1\right)\right\}$. Also in (3) the term

$$
C(\phi, a, \hbar) \equiv \frac{1}{2} \hbar^{2} \sum_{k} \mathcal{F}_{k}(\phi) a^{n}
$$

represents the still-to-be-chosen counterterm.

Besides the lattice action, we enlist the help of the associated lattice Hamiltonian as well as the ground state of that Hamiltonian in our search for a suitable counterterm and pseudofree theory. Assuming that the spacetime volume $V<\infty$, it is clear that full spacetime averages such as $\left\langle\left[\sum_{k} \phi_{k}^{r} a^{n}\right]^{p}\right\rangle$ are finite, for all positive integers $r$ and $p$, provided that all the corresponding sharp-time, spatial averages $\left\langle\left[\sum_{k}^{\prime} \phi_{k}^{r} a^{s}\right]^{p}\right\rangle$ are finite; for a proof, see [4]. In turn, for a large Euclidean time, the latter expression can be represented as

$$
\left\langle\left[\sum_{k}^{\prime} \phi_{k}^{r} a^{s}\right]^{p}\right\rangle=\int\left[\sum_{k}^{\prime} \phi_{k}^{r} a^{s}\right]^{p} \Psi(\phi)^{2} \Pi_{k}^{\prime} d \phi_{k},
$$

where $\Psi(\phi)$ denotes the ground state of the system.

Our interest next turns to an analysis of the putative ground state. 


\section{Choice of Counterterm}

To understand our basic approach, let us first consider the idealized example of a free-theory, Gaussian ground-state distribution

$$
\Psi_{G}(\phi)^{2} \equiv R e^{-A \Sigma_{k}^{\prime} \phi_{k}^{2} a^{s}}
$$

where $A=O(1)$, and, for integral $p \geq 0$, let us focus on the integrals

$$
I_{p}(A) \equiv R \int\left[\Sigma_{k}^{\prime} \phi_{k}^{2} a^{s}\right]^{p} e^{-A \Sigma_{k}^{\prime} \phi_{k}^{2} a^{s}} \Pi_{k}^{\prime} d \phi_{k}
$$

Such integrals can be evaluated exactly, but we prefer to study them in an approximate sense by steepest descent methods. To that end we introduce hyper-spherical coordinates [5] defined by

$$
\begin{gathered}
\phi_{k} \equiv \kappa \eta_{k}, \quad \Sigma_{k}^{\prime} \phi_{k}^{2}=\kappa^{2}, \quad \Sigma_{k}^{\prime} \eta_{k}^{2}=1, \\
0 \leq \kappa<\infty, \quad-1 \leq \eta_{k} \leq 1
\end{gathered}
$$

and it follows that

$$
I_{p}(A)=2 R \int \kappa^{2 p} a^{s p} e^{-A \kappa^{2} a^{s}} \kappa^{\left(N^{\prime}-1\right)} d \kappa \delta\left(1-\Sigma_{k}^{\prime} \eta_{k}^{2}\right) \Pi_{k}^{\prime} d \eta_{k}
$$

A steepest descent argument leads to

$$
I_{p}(A)=O\left(\left(N^{\prime} / A\right)^{p}\right) I_{0}(A)
$$

and a perturbation series for $I_{1}(A)$ about $I_{1}(1)$ is given by

$$
I_{1}(A)=I_{1}(1)-\Delta I_{2}(1)+\frac{1}{2} \Delta^{2} I_{3}(1)-\cdots,
$$

where $\Delta=A-1$. As $N^{\prime} \rightarrow \infty$, such a series has higher-order, termby-term divergences because the support of the ground-state distribution is concentrated on disjoint sets for distinct $A$ values due, specifically, to the factor $\kappa^{\left(N^{\prime}-1\right)}$ in the integrand. Our goal is to introduce a counterterm that effectively cancels the factor $\kappa^{\left(N^{\prime}-1\right)}$, and this can be accomplished, loosely speaking, by choosing an idealized example of a pseudofree model, about which to expand, with a ground-state distribution such that

$$
\Psi_{I}(\phi)^{2} \propto \kappa^{-\left(N^{\prime}-1\right)} e^{-A \Sigma_{k}^{\prime} \phi_{k}^{2} a^{s}}
$$


Observe that the use of the distribution $\Psi_{I}(\phi)^{2}$ in place of $\Psi_{G}(\phi)^{2}$ above leads to a series analogous to (12) that is term-by-term finite.

Naturally, there are many ways to choose a pseudofree ground state that has the desired property expressed in (13), and different models will require different versions. In fact, (13) has been the starting point to rapidly solve ultralocal models, which are nonrenormalizable quantum field theories without spatial derivatives having a vast symmetry that has been crucial to finding their solution previously [6]. But ultralocal models are not the subject of this article.

To deal with relativistic models, we focus on a ground state for the pseudofree ( $p f)$ model given by

$$
\Psi_{p f}(\phi)=K \frac{e^{-\Sigma_{k, l}^{\prime} \phi_{k} A_{k-l} \phi_{l} a^{2 s} / 2 \hbar-W\left(\phi a^{(s-1) / 2} / \hbar^{1 / 2}\right) / 2}}{\Pi_{k}^{\prime}\left[\Sigma_{l}^{\prime} J_{k, l} \phi_{l}^{2}\right]^{\left(N^{\prime}-1\right) / 4 N^{\prime}}} ;
$$

we discuss the constants $A_{k-l}$ and $J_{k, l}$ and the function $W$ below. This form for the ground state is ensured if we define the pseudofree theory - the theory about which a perturbation expansion is to take place - as

$$
\begin{gathered}
S_{p f}(h)=M_{p f} \int \exp \left[Z^{-1 / 2} \Sigma_{k} h_{k} \phi_{k} a^{n} / \hbar-\frac{1}{2} \sum_{k} \sum_{k^{*}}\left(\phi_{k^{*}}-\phi_{k}\right)^{2} a^{n-2} / \hbar\right. \\
\left.-\frac{1}{2} \hbar \sum_{k} \mathcal{F}_{k}(\phi) a^{n}\right] \Pi_{k} d \phi_{k}
\end{gathered}
$$

and choose $\mathcal{F}_{k}(\phi)$ to yield the denominator in (14). To make this connection, we appeal to the associated lattice Hamiltonian for the pseudofree model,

$$
\begin{aligned}
\mathcal{H}_{p f}= & -\frac{1}{2} \hbar^{2} a^{-s} \sum_{k}{ }_{k} \frac{\partial^{2}}{\partial \phi_{k}^{2}}+\frac{1}{2} \sum_{k}^{\prime} \sum_{k^{*}}^{\prime}\left(\phi_{k^{*}}-\phi_{k}\right)^{2} a^{s-2} \\
& +\frac{1}{2} \hbar^{2} \sum_{k}{ }^{\prime} \mathcal{F}_{k}(\phi) a^{s}-E_{0},
\end{aligned}
$$

and from this association we find that

$$
\begin{aligned}
\mathcal{F}_{k}(\phi) \equiv \frac{1}{4} & \left(\frac{N^{\prime}-1}{N^{\prime}}\right)^{2} a^{-2 s} \sum_{r, t}^{\prime} \frac{J_{r, k} J_{t, k} \phi_{k}^{2}}{\left[\Sigma_{l}^{\prime} J_{r, l} \phi_{l}^{2}\right]\left[\Sigma_{m}^{\prime} J_{t, m} \phi_{m}^{2}\right]} \\
& -\frac{1}{2}\left(\frac{N^{\prime}-1}{N^{\prime}}\right) a^{-2 s} \sum_{t}^{\prime} \frac{J_{t, k}}{\left[\Sigma_{m}^{\prime} J_{t, m} \phi_{m}^{2}\right]} \\
& +\left(\frac{N^{\prime}-1}{N^{\prime}}\right) a^{-2 s} \sum_{t}^{\prime} \frac{J_{t, k}^{2} \phi_{k}^{2}}{\left[\sum_{m}^{\prime} J_{t, m} \phi_{m}^{2}\right]^{2}} .
\end{aligned}
$$


Irrespective of the choice for $J_{k, l}$, we note that: (i) the denominator in the expression for the pseudofree ground state specifically leads to the counterterm in the Hamiltonian; (ii) the term in the exponent quadratic in $\phi$ is chosen to yield the spatial-gradient term in the Hamiltonian (and possibly part of $E_{0}$ ), and this requires that $A_{k-l}=O\left(a^{-(s+1)}\right)$; and (iii) the unspecified term $W$ ensures that no additional terms (other than the rest of $E_{0}$ ) appear in the Hamiltonian. The functional form of the argument in $W$ follows from the manner in which both $\hbar$ and $a$ appear in the Hamiltonian. In addition, note that the quadratic and denominator terms in $\Psi_{p f}(\phi)$ are correct for very large and very small field values, respectively; hence $W$ is relatively most effective for intermediate field values.

The choice $J_{k, l}=\delta_{k, l}$ leads to a local covariant potential for which $\mathcal{F}_{k}(\phi) \propto 1 / \phi_{k}^{2}$, but it also gives rise to a ground-state distribution with incipient normalization divergences at $\phi_{k}=0$, for each $k$, as $N^{\prime} \rightarrow \infty$. This behavior is appropriate for an ultralocal model, but not for a relativistic model. To overcome that feature, we choose the factors $J_{k, l}$ to provide a minimally regularized, lattice-symmetric, local spatial averaging in the form

$$
J_{k, l} \equiv \frac{1}{2 s+1} \delta_{k, l \in\left\{k \cup k_{n n}\right\}}
$$

where $\delta_{k, l}$ is a Kronecker delta. This notation means that an equal weight of $1 /(2 s+1)$ is given to the $2 s+1$ points in the set composed of $k$ and its $2 s$ nearest neighbors in the spatial sense only; $J_{k, l}=0$ for all other points in that spatial slice. [Specifically, we define $J_{k, l}=1 /(2 s+1)$ for the points $l=k=$ $\left(k_{0}, k_{1}, k_{2}, \ldots, k_{s}\right), l=\left(k_{0}, k_{1} \pm 1, k_{2}, \ldots, k_{s}\right), l=\left(k_{0}, k_{1}, k_{2} \pm 1, \ldots, k_{s}\right), \ldots$, $l=\left(k_{0}, k_{1}, k_{2}, \ldots, k_{s} \pm 1\right)$.] This definition implies that $\Sigma_{l}^{\prime} J_{k, l}=1$.

In the continuum limit, it is important to observe that the form of the counterterm given by (17) leads to a local covariant potential, albeit an unconventional one.

\section{The Continuum Limit, and Term-by-term Finiteness of a Perturbation Analysis}

Before focusing on the limit $a \rightarrow 0$ and $L \rightarrow \infty$, we note several important facts about ground-state averages of the direction field variables $\left\{\eta_{k}\right\}$. First, we assume that such averages have two important symmetries: (i) averages 
of an odd number of $\eta_{k}$ variables vanish, i.e.,

$$
\left\langle\eta_{k_{1}} \cdots \eta_{k_{2 p+1}}\right\rangle=0
$$

and (ii) such averages are invariant under any spacetime translation, i.e.,

$$
\left\langle\eta_{k_{1}} \cdots \eta_{k_{2 p}}\right\rangle=\left\langle\eta_{k_{1}+l} \cdots \eta_{k_{2 p}+l}\right\rangle
$$

for any $l \in \mathbb{Z}^{n}$ due to a similar translational invariance of the lattice Hamiltonian. Second, we note that for any ground-state distribution, it is necessary that $\left\langle\eta_{k}^{2}\right\rangle=1 / N^{\prime}$ for the simple reason that $\Sigma_{k}^{\prime} \eta_{k}^{2}=1$. Hence, $\left|\left\langle\eta_{k} \eta_{l}\right\rangle\right| \leq 1 / N^{\prime}$ as follows from the Schwarz inequality. Since $\left\langle\left[\Sigma_{k}^{\prime} \eta_{k}^{2}\right]^{2}\right\rangle=1$, it follows that $\left\langle\eta_{k}^{2} \eta_{l}^{2}\right\rangle=O\left(1 / N^{\prime 2}\right)$. Similar arguments show that for any ground-state distribution

$$
\left\langle\eta_{k_{1}} \cdots \eta_{k_{2 p}}\right\rangle=O\left(1 / N^{\prime p}\right)
$$

which will be useful in the sequel.

In discussing the moments below, we remind the reader [see the discussion regarding (6)] that if sharp time averages are made finite, then the corresponding spacetime averages in the distribution determined by the lattice action will also be finite.

\section{Field strength renormalization}

For $\left\{h_{k}\right\}$ a suitable spatial test sequence, we insist that expressions such as

$$
\int Z^{-p}\left[\Sigma_{k}^{\prime} h_{k} \phi_{k} a^{s}\right]^{2 p} \Psi_{p f}(\phi)^{2} \Pi_{k}^{\prime} d \phi_{k}
$$

are finite in the continuum limit. Due to the intermediate field relevance of the factor $W$ in the pseudofree ground state, an approximate evaluation of the integral (22) will be adequate for our purposes. Thus, we are led to consider

$$
\begin{aligned}
K \int Z^{-p}\left[\Sigma_{k}^{\prime} h_{k} \phi_{k} a^{s}\right]^{2 p} \frac{e^{-\Sigma_{k, l}^{\prime} \phi_{k} A_{k-l} \phi_{l} a^{2 s} / \hbar-W}}{\Pi_{k}^{\prime}\left[\Sigma_{l}^{\prime} J_{k, l} \phi_{l}^{2}\right]^{\left(N^{\prime}-1\right) / 2 N^{\prime}}} \Pi_{k}^{\prime} d \phi_{k} \\
\simeq 2 K_{0} \int Z^{-p} \kappa^{2 p}\left[\Sigma_{k}^{\prime} h_{k} \eta_{k} a^{s}\right]^{2 p} \\
\times \frac{e^{-\kappa^{2} \Sigma_{k, l}^{\prime} \eta_{k} A_{k-l} \eta_{l} a^{2 s} / \hbar}}{\Pi_{k}^{\prime}\left[\Sigma_{l}^{\prime} J_{k, l} \eta_{l}^{2}\right]^{\left(N^{\prime}-1\right) / 2 N^{\prime}}} d \kappa \delta\left(1-\Sigma_{k}^{\prime} \eta_{k}^{2}\right) \Pi_{k}^{\prime} d \eta_{k},
\end{aligned}
$$


where $K_{0}$ is the normalization factor when $W$ is dropped. Our goal is to use this integral to determine a value for the field strength renormalization constant $Z$. To estimate this integral we first replace two factors with $\eta$ variables by their appropriate averages. In particular, the quadratic expression in the exponent is estimated by

$$
\kappa^{2} \Sigma_{k, l}^{\prime} \eta_{k} A_{k-l} \eta_{l} a^{2 s} \simeq \kappa^{2} \Sigma_{k, l}^{\prime} N^{\prime-1} A_{k-l} a^{2 s} \propto \kappa^{2} N^{\prime} a^{2 s} a^{-(s+1)},
$$

and the expression in the integrand is estimated by

$$
\left[\Sigma_{k}^{\prime} h_{k} \eta_{k} a^{s}\right]^{2 p} \simeq N^{\prime-p}\left[\Sigma_{k}^{\prime} h_{k} a^{s}\right]^{2 p} .
$$

The integral over $\kappa$ is then estimated by first rescaling the variable $\kappa^{2} \rightarrow$ $\kappa^{2} /\left(N^{\prime} a^{s-1} / \hbar\right)$, which then leads to an overall integral estimate proportional to

$$
Z^{-p}\left[N^{\prime} a^{s-1}\right]^{-p} N^{\prime-p}\left[\Sigma_{k}^{\prime} h_{k} a^{s}\right]^{2 p}
$$

at this point, all factors of $a$ are now outside the integral. For this result to be meaningful in the continuum limit, we are led to choose $Z=N^{\prime-2} a^{-(s-1)}$. However, $Z$ must be dimensionless, so we introduce a fixed positive quantity $q$ with dimensions of an inverse length, which allows us to set

$$
Z=N^{\prime-2}(q a)^{-(s-1)} \text {. }
$$

\section{Mass and coupling constant renormalization}

A power series expansion of the mass and coupling constant terms in the full spacetime distribution leads to two kind of moments given by

$$
\left\langle\left[m_{0}^{2} \Sigma_{k} \phi_{k}^{2} a^{n}\right]^{p}\right\rangle, \quad\left\langle\left[\lambda_{0} \Sigma_{k} \phi_{k}^{4} a^{n}\right]^{p}\right\rangle,
$$

for $p \geq 1$, which we treat together as part of the larger family governed by $\left\langle\left[g_{0, r} \Sigma_{k} \phi_{k}^{2 r} a^{n}\right]^{p}\right\rangle$ for integral $r \geq 1$. We deal with these spacetime moments by considering analogous sharp time moments given by

$$
\begin{aligned}
K \int\left[g_{0, r} \Sigma_{k}^{\prime} \phi_{k}^{2 r} a^{s}\right]^{p} \frac{e^{-\Sigma_{k, l}^{\prime} \phi_{k} A_{k-l} \phi_{l} a^{2 s} / \hbar-W}}{\Pi_{k}^{\prime}\left[\Sigma_{l}^{\prime} J_{k, l} \phi_{l}^{2}\right]^{\left(N^{\prime}-1\right) / 2 N^{\prime}}} \Pi_{k}^{\prime} d \phi_{k} \\
\simeq 2 K_{0} \int g_{0, r}^{p} \kappa^{2 r p}\left[\Sigma_{k}^{\prime} \eta_{k}^{2 r} a^{s}\right]^{p} \\
\quad \times \frac{e^{-\kappa^{2} \Sigma_{k, l}^{\prime} \eta_{k} A_{k-l} \eta_{l} a^{2 s} / \hbar}}{\Pi_{k}^{\prime}\left[\Sigma_{l}^{\prime} J_{k, l} \eta_{l}^{2}\right]^{\left(N^{\prime}-1\right) / 2 N^{\prime}}} d \kappa \delta\left(1-\Sigma_{k}^{\prime} \eta_{k}^{2}\right) \Pi_{k}^{\prime} d \eta_{k} .
\end{aligned}
$$


The quadratic exponent is again estimated as

$$
\kappa^{2} \Sigma_{k, l}^{\prime} \eta_{k} A_{k-l} \eta_{l} a^{2 s} \propto \kappa^{2} N^{\prime} a^{2 s} a^{-(s+1)},
$$

while the integrand factor

$$
\left[\Sigma_{k}^{\prime} \eta_{k}^{2 r}\right]^{p} \simeq N^{\prime p} N^{\prime-r p} .
$$

The same transformation of variables used above precedes the integral over $\kappa$, and the result is an integral, no longer depending on $a$, that is proportional to

$$
g_{0, r}^{p} N^{\prime-(r-1) p} a^{s p} / N^{\prime r p} a^{(s-1) r p} .
$$

To have an acceptable continuum limit, it suffices that

$$
g_{0, r}=N^{\prime(2 r-1)}(q a)^{(s-1) r-s} g_{r},
$$

where $g_{r}$ may be called the physical coupling factor. Moreover, it is noteworthy that $Z^{r} g_{0, r}=\left[N^{\prime}(q a)^{s}\right]^{-1} g_{r}$, for all values of $r$, which for a finite spatial volume $V^{\prime}=N^{\prime} a^{s}$ leads to a finite nonzero result for $Z^{r} g_{0, r}$. It should not be a surprise that there are no divergences for all such interactions because the source of all divergences has been neutralized!

We may specialize the general result established above to the two cases of interest to us. Namely, when $r=1$ this last relation implies that $m_{0}^{2}=$ $N^{\prime}(q a)^{-1} m^{2}$, while when $r=2$, it follows that $\lambda_{0}=N^{\prime 3}(q a)^{s-2} \lambda$. In these cases it also follows that $Z m_{0}^{2}=\left[N^{\prime}(q a)^{s}\right]^{-1} m^{2}$ and $Z^{2} \lambda_{0}=\left[N^{\prime}(q a)^{s}\right]^{-1} \lambda$, which for a finite spatial volume $V^{\prime}=N^{\prime} a^{s}$ leads to a finite nonzero result for $Z m_{0}^{2}$ and $Z^{2} \lambda_{0}$, respectively.

\section{Conclusion}

For scalar nonrenormalizable quantum field models, we have shown that the choice of an unconventional counterterm, but one that is proportional to $\hbar^{2}$ and therefore nonclassical, leads to a formulation for which a perturbation analysis for both the mass term and the nonlinear interaction term of the full spacetime averages, expanded about the appropriate pseudofree model, are term-by-term finite. Thanks to the unconventional counterterm, it is noteworthy that additive renormalization has been everywhere replaced by 
multiplicative renormalization. A natural question to ask is how the cancelation of the factor $\kappa^{\left(N^{\prime}-1\right)}$ in (10) relates to the classical hard core observation regarding fields such as $\phi_{\text {singular }}$ given following (1); our answer is to observe that the existence of the classical hard core tends to suggest there may a hard core in the quantum theory as well, but as in all functional integrals over fields, the set of fields in the classical domain makes a contribution in the quantum theory of measure zero.

Alternative insight into such models may possibly be obtained by Monte Carlo studies of the full, nonperturbative model including the special counterterm; for a preliminary discussion of such an approach, see [7].

\section{References}

[1] M. Aizenman, "Proof of the Triviality of $\varphi_{d}^{4}$ Field Theory and Some Mean-Field Features of Ising Models for $d>4$ ", Phys. Rev. Lett. 47, 14, E-886 (1981); J. Fröhlich, "On the Triviality of $\lambda \varphi_{d}^{4}$ Theories and the Approach to the Critical Point in $d \geq 4$ Dimensions", Nuclear Physics $B$ 200, 281-296 (1982).

[2] J.R. Klauder, Beyond Conventional Quantization, (Cambridge University Press, Cambridge, 2000 \& 2005).

[3] J.R. Klauder, "Continuous and Discontiuous Perturbations", Science 199, 735-740 (1978); see also "Rethinking Renormalization", arXiv:0904.2869.

[4] J.R. Klauder, "A New Approach to Nonrenormalizable Models", Ann. Phys. 322, 2569-2602 (2007).

[5] J.R. Klauder, "Poisson Distributions for Sharp-Time Fields: Antidote for Triviality", hep-th/9511202, pp 22-28; J.R. Klauder, "Isolation and Expulsion of Divergences in Quantum Field Theory", Int. J. Mod. Phys. B 10, 1473-1483 (1996).

[6] J.R. Klauder, "Rethinking Renormalization", arXiv:0904.2869.

[7] J.R. Klauder, "Divergence-free Nonrenormalizable Models", J. Phys. A: Math. Theor. 41, 335206 (15pp) (2008). 